\title{
Pflanzenschutz: Neue Gesetze und ihr Nutzen
}

\author{
Herbert Resseler · Otto Klein • Manfred Lefèvre
}

Erhalten: 14. Oktober 2009/Akzeptiert: 19. Oktober 2009/Online veröffentlicht: 18. November 2009

(C) Springer-Verlag 2009

Zusammenfassung Die Diskussionen im Rahmen der Novellierung der EU-Richtlinie 91/414/EEC sind oft von unsachlichen Angriffen auf das bestehende Zulassungsverfahren für Pflanzenschutzmittel und die beteiligten Behörden geprägt. Das hat die Verbraucher verunsichert und den Eindruck in der Öffentlichkeit verstärkt, das Zulassungssystem in Europa sei mangelhaft und die Gesundheit der Verbraucher sowie die Umwelt wären durch Pflanzenschutzmittelrückstände gefährdet. Eine genaue Überprüfung dieser Meldungen bestätigt jedoch, dass das System zuverlässig arbeitet und aktuelle wissenschaftliche Erkenntnisse berücksichtigt. Das für aquatische und terrestrische Organismen berechnete Risikopotenzial in Deutschland ist seit 1987 kontinuierlich zurückgegangen. Vor diesem Hintergrund werden die Auswirkungen und der Nutzen der neuen gesetzlichen Regelungen diskutiert, deren Komplexität ein noch nicht da gewesenes Ausmaß erreicht hat.

Aus Sicht der Industrie sind überschaubare gesetzliche Regelungen und praktikable Ausführungsbestimmungen unabdingbar, um die Planbarkeit und Rechtssicherheit für die Entwicklung von Produkten zu gewährleisten.

Schlüsselwörter Chemischer Pflanzenschutz .

EU-Pflanzenschutzrecht · EU-Richtlinie 91/414/EEC · Gewässer $\cdot$ Handlungsalternativen · Integrierter Pflanzenschutz $\cdot$ Lebensmittel $\cdot$ Novellierung $\cdot$ Ökologischer Landbau $\cdot$ Risiken $\cdot$ Risikoindikatoren $\cdot$ Rückstände $\cdot$ Schutzziel

Verantwortlicher Herausgeber: Henning Friege

Im Rahmen der Beitragsserie „Regulatorische Ökotoxikologie“, Hrsg. Tobias Frische, Jan Ahlers, Bettina Hitzfeld

H. Resseler $(\bowtie) \cdot$ O. Klein $\cdot$ M. Lefèvre Industrieverband Agrar e. V.,

Mainzer Landstraße 55, 60329 Frankfurt am Main, Deutschland

E-Mail: herbert.resseler@syngenta.com
Plant protection: New legislations and their benefit

Abstract The debate about the revision of the EU directive $91 / 414$ has often been characterized by unobjective criticism of the existing regulations and of the authorities involved in the regulatory procedure. This made many consumers feel uncertain and left the public with the impression that the current regulatory system for plant protection products in Europe was not safe for consumers and the environment. However, a detailed evaluation of these statements has clearly demonstrated that the current regulatory procedure is safe and recent scientific knowledge is taken into account. The risk potential calculated for aquatic and terrestrial organisms in Germany has continuously decreased since 1987.

The effects and the benefits of the new regulations, which have reached a level of complexity as never before, are discussed in this context.

From an industry perspective, clear legal regulations and practical implementations are essential to guarantee the transparence of the regulatory process and predictability of the outcome.

Keywords Chemical plant protection - Choice of action EU Directive 91/414/EEC · EU Plant Protection Regulation . Food · Integrated pest management $\cdot$ Organic farming . Protection goal $\cdot$ Residues $\cdot$ Revision · Risk indicators · Risks $\cdot$ Water

\section{Einleitung und Problemstellung}

Der Nutzen des chemischen Pflanzenschutzes ist im Bewusstsein der Öffentlichkeit in Europa wenig präsent. Erst 2008 wurde aufgrund zeitweise stark gestiegener Preise 
für einige Nahrungsmittel vermehrt wahrgenommen, dass Bevölkerungswachstum und gestiegene Nachfrage über Jahre hinweg zu historisch niedrigen Weltgetreidevorräten geführt haben. Die pro Kopf verfügbare Ackerfläche schrumpft. Die Anzahl der Hungernden auf der Welt ist nach UN/FAO-Angaben weiter gestiegen und nähert sich der Milliardengrenze. Diese Trends setzen sich augenscheinlich langfristig fort. Deutschland hat nach Abzug der importierten Nahrungs- und Futtermittel einen Selbstversorgungsgrad von nur $82 \%$. Solchen Informationen wird bei uns wenig Aufmerksamkeit geschenkt, da in Deutschland Ernährungssicherheit herrscht und der Anteil des Einkommens, der für Nahrungsmittel aufgewendet werden muss, auf etwa $11 \%$ gesunken ist. Vor diesem Hintergrund wird im Zusammenhang mit chemischen Pflanzenschutzmaßnahmen mehr über deren potenzielle Risiken als über deren Nutzen zur Sicherstellung der globalen Nahrungsmittelversorgung diskutiert. Ohne Pflanzenschutz würden die möglichen Ernteverluste in Westeuropa bei etwa $60 \%$ liegen, weltweit bei $40 \%$ (Oerke und Dehne 1997). Mit anderen Worten: Das heutige, ohnehin „knappe“ Produktionsniveau könnte nur um den Preis eines deutlich höheren Flächen- und Naturverbrauchs aufrechterhalten werden (Kirchmann et al. 2009).

\section{Pflanzenschutzmittelzulassung - ein komplexer Vorgang}

Pflanzenschutzmittel und deren Anwendungen werden ähnlich wie Arzneimittelwirkstoffe, Lebensmittelzusatzstoffe oder Biozide umfangreichen Prüfungen unterworfen. Voraussetzung für die Zulassung eines Pflanzenschutzmittels ist zunächst, dass die in ihm enthaltenen Wirkstoffe auf EU-Ebene von der europäischen Zulassungsbehörde EFSA und den nationalen Zulassungsbehörden der Mitgliedsstaaten bewertet und von der EU-Kommission in Zusammenarbeit mit den Mitgliedsländern in die Positivliste (Anhang I) der Richtlinie 91/414/EEC aufgenommen wurden (http:// ec.europa.eu/food/plant/protection/evaluation/dir91-414 eec_en.htm). Die Zulassung der Handelsprodukte obliegt dann in einem zweiten Schritt den einzelnen Mitgliedsstaaten. Im Zulassungsverfahren in Deutschland arbeitet das Bundesamt für Verbraucherschutz und Lebensmittelsicherheit (BVL 2009a,b,c,d) mit drei weiteren Behörden zusammen, und zwar dem Julius Kühn-Institut (JKI, vormals BBA, http://www.jki.bund.de/, Prüfung von Wirksamkeit und Nutzen der Mittel (BVL 2004)), dem Bundesinstitut für Risikobewertung (BfR, http://www.bfr.bund.de/cd/240, Bewertung der gesundheitlichen Risiken für Verbraucher und Anwender, Vorschläge zur Festsetzung europäischer Rückstandshöchstmengen) sowie dem Umweltbundesamt (UBA, Beurteilung im Hinblick auf mögliche Auswirkungen auf den Naturhaushalt, http://www.umweltbundesamt.de/ chemikalien/pflanzenschutz.htm).

\section{Gefahrenbewertung anstelle von Risikobewertung}

Führende internationale Zulassungsbehörden wie EFSA und die US-EPA bewerten (bisher) nach dem expositionsbasierten Ansatz (s. u.). Diese Vorgehensweise wird nach der Novellierung in der EU durch den gefahrenbasierten Ansatz ergänzt. Dass dieser Schritt auch bei den Experten auf Behördenseite nicht unumstritten ist, zeigt die Position des BfR: Das BfR hat sich klar für die Beibehaltung der expositionsbasierten Risikobewertung für die Zulassung von Pflanzenschutzmitteln ausgesprochen (BfR 2008b). Greim (2007, S. 86) bewertet gefahrenbasierte Klassifizierung und Kennzeichnung von Produkten als „nicht mehr dem Stand der wissenschaftlichen Erkenntnis" entsprechend.

Expositionsorientierter Ansatz: Risiko ist eine Funktion der Wahrscheinlichkeit des Eintritts eines Schadens und der Schadenshöhe (= Gefahr) nach seinem Eintritt (d.h. Risiko $=$ Gefahr $\times$ Eintrittswahrscheinlichkeit). Durch quantitative Expositionsabschätzung orientiert sich die Produktbewertung bisher nicht nur am Gefährdungspotenzial eines Wirkstoffs in hochdosierter Form, sondern auch an der Dosis, die tatsächlich zu erwarten ist. Die Erkenntnis von Paracelsus $(, \ldots$ allein die Dosis macht, dass ein Ding kein Gift ist") wird berücksichtigt. Pflanzenschutzmittel werden nur zugelassen, wenn ihre Anwendung sicher für Mensch und Umwelt ist. Mit der zunehmenden Etablierung von gefahrenbedingten Ausschlusskriterien wird nun der Eindruck vermittelt, die Zulassungsbehörden hätten bisher Pflanzenschutzmittelanwendungen zugelassen, die ,unsicher" waren und Mensch und Umwelt schädigten. Dass Sicheres noch sicherer gemacht werden sollte, ist schwer zu vermitteln, da es einen inneren Widerspruch darstellt.

\section{Vergleichende Bewertung von Produkten}

Es gibt bisher keinerlei Ausführungsbestimmungen darüber, wie die „vergleichende Bewertung“ von Produkten durchzuführen ist, die nun vom Gesetzgeber verlangt wird. Potenzielle Risiken und Nutzen im Anwendungsbereich eines Produktes müssen denen alternativer Produkte gegenüber gestellt werden. Die begrenzte Erfahrung, die man auf diesem Gebiet z.B. in Schweden hat, ist nicht ohne weiteres auf andere Länder und das Zusammenspiel in der EU übertragbar. Die pflanzenbaulichen Probleme sind in den südlichen Mitgliedsstaaten ganz anders gelagert als z. B. in einem skandinavischen Land. Auch ist ungeklärt, wie unterschiedliche Problembereiche gegeneinander abgewogen werden, d.h. wie die Toxizität eines Wirkstoffes gegenüber 
z.B. Fischen mit der Toxizität des zu vergleichenden Wirkstoffes z. B. gegenüber Vögeln verrechnet wird. Es erscheint wissenschaftlich fragwürdig, Risikoindizes und TER-Werte (Toxicity/Exposure Ratio) über verschiedene Speziesgrenzen hinweg (z. B. für Algen, Fische, Regenwürmer, Vögel, Nager und Säugetiere) zu aggregieren. Administrativer Aufwand, wissenschaftliches Ergebnis und Nutzen einer derartigen vergleichenden Bewertung erscheinen höchst dubios. Die Industrie muss sogar noch einen Schritt weiter gehen: Sie muss das Ergebnis der Vergleichenden Bewertung in mehreren Teilmärkten der EU prognostizieren, um abzuschätzen, ob die Entwicklung und Einführung des Produkts für die EU lohnt. Die Behörden werden auch weiterhin die Produkte hinsichtlich ihrer Wirkung auf Mensch und Umwelt kritisch prüfen und sichere Produktanwendungen zulassen. Darüber hinausgehende Bestrebungen (,sicherer als sicher") sind wissenschaftlich fragwürdig. Die quantitative Nutzenanalyse sollte dem Marktgeschehen überlassen werden.

\section{Risikoreduktion - ein historisches Ziel}

Laut dem nationalen Aktionsplan zum nachhaltigen Einsatz von Pflanzenschutzmitteln ist für den Zeitraum von 1987 bis 2006 das Risikopotenzial für Gewässerorganismen zurückgegangen (BMELV 2008). Für Herbizide wird eine Senkung des akuten Risikopotenzials auf etwa $50 \%$, für Fungizide auf etwa $70 \%$ und für Insektizide auf ca. $20 \%$ verzeichnet. Im Bereich Terrestrik wurde eine vergleichbare Tendenz festgestellt. Hinter dieser Reduktion stecken zahlreiche Innovationen und Optimierungen, u. a. durch die Entwicklung selektiver wirkender Mittel, bei der Gerätetechnik und bei Prognoseverfahren. Vor diesem Hintergrund ist die Frage berechtigt, inwieweit über den oben festgestellten Trend der Risikoabnahme hinaus weitere Risikoreduzierungsmaßnahmen staatlicherseits erforderlich sind. Der Nutzen zusätzlicher Maßnahmen ist nicht ersichtlich. Die mögliche Folge wäre, dass für bestimmte Kulturen bedeutsame Produkte nicht mehr verfügbar sind.

Die belebte Umwelt, d.h. die Vielfalt von Tieren und Pflanzen sowie ihre Wechselwirkungen und Funktionen in einem Ökosystem, zeichnet sich durch ein Höchstmaß an Vielschichtigkeit aus. Es leben beispielsweise in Deutschland ca. 45.000 beschriebene Tierarten, ca. 30.000 davon sind allein Insekten. Pflanzenschutzmittel beeinflussen, ebenso wie jede andere landwirtschaftliche Maßnahme von Bodenbearbeitung bis Fruchtfolge - die Individuen in einer Kulturlandschaft (Küster 2009). Eine „,nebenwirkungsfreie Landwirtschaft" wird es deshalb nicht geben. Die Frage ist allerdings, ob die Population und das Ökosystem von der Maßnahme in unvertretbarer Weise beeinflusst werden. Dies zu prüfen ist Aufgabe der Risikobewertung in der Zulassung. Die in Bezug auf die verschiedenen Kom- partimente der belebten Umwelt (Gewässerorganismen, Bodenbewohner, Honigbienen, Nicht-Zielarthropoden, Nicht-Zielpflanzen, Vögel, Säugetiere) seitens der Industrie erstellten Risikobetrachtungen haben bereits in den vergangenen Jahren einen drastischen Anstieg an Komplexität erlebt. Es ist abzusehen, dass dieser Trend sich vor dem Hintergrund der neuen legislativen Vorgaben fortsetzt oder sich in manchen Arbeitsgebieten sogar noch verstärkt. Dies wäre noch verständlich, wenn eine maßgebliche Erhöhung des Schutzniveaus für die Organismen erreicht würde - dafür aber fehlen fundierte Belege. Als Beispiel für diesen sehr verkürzt dargestellten Zusammenhang seien u. a. die Revision der Risikobetrachtung für Vögel und Säuger (EFSA 2008) sowie für Bodenorganismen genannt.

Das wissenschaftliche Interesse an weiterer Klärung von Zusammenhängen und Effekten ist ohne Zweifel berechtigt, der Stand der Kenntnisse entwickelt sich laufend weiter. Vor einer Umsetzung von Forschungsergebnissen in die Zulassungspraxis bedarf es aber einer Überprüfung, ob das zur Lösung anstehende Thema überhaupt als Problem im eingangs genannten Kontext der Kulturlandschaft existiert (,reality check"). Wissenschaftler, die ihre aktuellen Forschungsergebnisse mit einer Änderung des Zulassungsverfahrens verbunden wissen wollen, sollten nachweisen, ob das Schutzgut tatsächlich gefährdet ist und welcher konkreter Nutzen durch das Projekt in Bezug auf eine erhöhte Sicherheit des Schutzgutes zu erwarten ist. Unterbleiben diese Schritte, ist mit einem hohen $\mathrm{Ma}$ an Unsicherheit und Aufwand in Bezug auf nationale und europaweite Zulassungen zu rechnen, ohne dass ein Nutzen in Bezug auf eine erhöhte Sicherheit des Schutzgutes zu erkennen wäre.

\section{Kritische Themenfelder - eine Analyse}

\subsection{Rückstände in Lebensmitteln}

Das BVL berichtet, dass 97,3\% der in Deutschland 2007 produzierten Ware an Obst und Gemüse die gesetzlichen Höchstgehalte einhält (risikoorientierte Probenahme, 2,7\% Überschreitungen bei D-Ware, EU-Ware: $5 \%$, Nicht EUWare 9,5\%; BVL 2009e,f). Bezieht man die anderen landwirtschaftlichen Erzeugnisse mit ein, so ist der Anteil an Proben unterhalb der Höchstgehalte noch größer. Das BfR konnte auch nicht die von Greenpeace und PAN beschriebenen - angeblich durch die Rückstände von chemisch-synthetischen Pflanzenschutzmitteln bedingten - gesundheitlichen Risiken bei Wein feststellen (BfR 2008 $\mathrm{c}^{1}$ ); die Debatte

\footnotetext{
${ }^{1}$ Die Rückstandskonzentrationen bewegten sich weit unterhalb einer akut oder chronisch toxischen Schädigungsmöglichkeit $(<10 \%$ der Akuten Referenzdosis (ARfD)- und/oder Acceptable Daily Intake (ADI)-Wert-Ausschöpfung, BfR 2008c)
} 
wurde bis in das EU-Parlament getragen. Die Höchstgehalte werden vom BfR nach international anerkannten wissenschaftlichen Standards festgesetzt. In der Festlegung der toxikologischen Referenzwerte ADI und ARfD ist zudem eine beachtliche Sicherheitsmarge von in der Regel mindestens 100 enthalten. Geringfügige Überschreitungen der Höchstgehalte beeinträchtigen somit in keiner Weise die Verbrauchersicherheit. Trotz der Vielzahl von Anwendungen und Umweltbedingungen gibt es in $97 \%$ der Fälle ohnehin keine Überschreitung der Höchstmengen (BVL 2009e); eine Umstellung im Zulassungsverfahren würde daran vermutlich nichts ändern. Weitere Fortschritte auf diesem Gebiet sind eher durch technische Entwicklung (Applikationstechnik) und noch sensibleren Umgang mit den Produkten bei der Anwendung (Ausbildung und Beratung) zu erwarten. Die britische Zulassungsbehörde PSD kommentierte die zu erwartenden neuen gesetzlichen Regelungen der EU im November 2008: „No meaningful benefits to public health protection from any criteria have been demonstrated" (PSD 2008)

In jüngster Zeit jedoch wird der Aspekt der Vorsorge verstärkt in die Diskussion eingebracht. Die dabei zum Teil recht willkürlich formulierten Kriterien und Schutzziele werden in der öffentlichen Diskussion von beteiligten Nicht-Regierungsorganisationen als die (bessere) Alternative zu den EU-weit harmonisierten Bewertungsgrundsätzen dargestellt. Verknüpft wird dies mit teilweise massiver Kritik an der bisherigen Zulassungspraxis und der Sicherheit von Pflanzenschutzmitteln (u. a. in: Greenpeace 2002; Greenpeace und Global 2000 2008; Krautter 2009). Derartige Meldungen führen zu einer starken Verunsicherung bei Verbrauchern, Handel und Politikern. Besonders im Hinblick auf die Verbrauchersicherheit hat die zuständige Fachbehörde, das Bundesamt für Risikobewertung (BfR), jedoch festgestellt, dass nach wissenschaftlicher Betrachtung kein Anlass zur Besorgnis besteht (BfR 2008d). Relevante Stellungnahmen sind auf der Internetseite des BfR einsehbar.

\subsection{Belastung von Grundwasser und Oberflächenwasser}

Das Versickerungspotenzial und damit das Risiko einer Kontamination des Grundwassers lässt sich im Zulassungsverfahren mithilfe von Simulationsmodellen und Lysimeterversuchen recht genau abschätzen. Ergebnisse aus solchen Untersuchungen werden im Rahmen des Zulassungsverfahrens gefordert. Hohe Konzentrationen im Oberflächenwasser sind demgegenüber häufig auf Fehlanwendungen, Unfälle und/oder Punktkontaminationen zurückzuführen, die nicht Gegenstand der Zulassungsprüfung sein können, da hier in aller Regel unsachgemäße Reinigung, Leckage in Abwasseranlagen oder Ausläufe aus Kläranlagen vorliegen.

\subsection{Grundwasser}

Eine Untersuchung der Meldungen zu Wirkstofffunden im Grundwasser in Deutschland zeigt, dass $94 \%$ aller Fundmeldungen, die der Industrie von den Zulassungsbehörden zur Aufklärung aufgegeben wurden, als nicht zulassungsrelevant bewertet werden müssen (Schmidt et al. 2005). Diese Funde gehen nicht auf eine bestimmungsgemäße und sachgerechte Anwendung von Pflanzenschutzmitteln in der Landwirtschaft zurück. Insgesamt waren 151 Fundmeldungen untersucht worden. Hauptursachen für die Fundmeldungen, wie die Überprüfungen ergaben, waren:

- Eintritt von Oberflächenwasser in die Messstelle (25\%),

- Einfluss von Abwasserleitungen und Sickergruben (17\%),

- Probenkontamination/Analysenfehler (13\%),

- mangelhafte Messstellenqualität (10\%),

- Abfallentsorgung (9\%) und

- kein Grundwasser (4\%).

Nur bei fünf Funden ( $3 \%$ aller Meldungen) konnte eine Versickerung von der behandelten Fläche nicht ausgeschlossen werden. In Anbetracht des breiten Einsatzes der untersuchten fünf Wirkstoffe und der hohen Untersuchungsdichte (2000 bis 4000 Messstellen jährlich im Zeitraum von 1990 bis 2002) ist diese Zahl möglicherweise zulassungsrelevanter Grenzwertüberschreitungen sehr gering. Die hohe Sicherheit des aktuellen Zulassungsverfahrens wird durch diese Fundaufklärung zum bundesweiten Grundwassermonitoring bestätigt. Die im Bericht des Sachverständigenrats für Umweltfragen (SRU 2004, S. 223 ff.) gezogene Schlussfolgerung, das Grundwasser sei unzureichend geschützt und das Zulassungsverfahren auch deswegen reformbedürftig, lässt sich nicht mit vor Ort verifizierten Befunden belegen. Offenbar wurden die Meldungen ungeprüft übernommen.

\subsection{Oberflächenwasser}

Geringe Einträge von Pflanzenschutzmitteln in Oberflächengewässer können nicht gänzlich ausgeschlossen werden, solange landwirtschaftlich genutzte Flächen in Oberflächengewässer „drainieren“. Es wird alles getan, um diese Einträge so gering wie möglich zu halten. Bei potenziell kritischen Substanzanwendungen verhängt die Zulassungsbehörde Abstandsauflagen. Die Industrie führt das europaweite Projekt „TOPPS“ (http://www.topps-life.org) zur Ausbildung und Sensibilisierung der Landwirte beim Umgang mit Pflanzenschutzmitteln und die nationale PAMIRAKampagne zur Entsorgung von restentleerten und gespülten Pflanzenschutzpackmitteln durch (http://www.pamira.de). Die Frachten zugelassener Pflanzenschutzmittel in Flüssen gehen seit Jahren zurück, wie Auswertungen von Pegelstationen an Rhein, Main und Ruhr zeigen (Bach 2008). Untersuchungen von Gutsche und Strassemeyer (2007) belegen, dass das Risikopotenzial für aquatische Organismen (wie 
auch für terrestrische) seit 1987 kontinuierlich zurückgeht. Flankierende Maßnahmen und Mittel, die im Rahmen der Novellierung weiterer EU-Vorgaben für Ausbildung und Beratung von Landwirten zur Verfügung gestellt werden, sind sehr begrüßenswert.

\section{Kaum Handlungsalternativen}

Neben der kontrovers diskutierten Frage, ob chemisch-synthetische Pflanzenschutzmittel „nur vermeintlich“ oder ,tatsächlich" sicher sind, sollte auch der Frage nachgegangen werden, welche Handlungsalternativen die Kritiker des integrierten Pflanzenschutzes anbieten. Vielfach wird die Umstellung auf den ,„ökologischen Landbau“ mit der Begründung vorgeschlagen, er ,verzichte auf chemische Pestizide und Düngemittel“". Neben nicht-chemischen Verfahren setzt aber auch der ökologische Landbau Pflanzenschutzmittel ein, um seine Ernten vor Pilzbefall und Insektenfraß zu schützen: Azadirachtin, verschiedene Bacillus thuringiensis-Stämme, Kupfer, Pyrethrine, Rotenon und Schwefel gehören zu den angewendeten Wirkstoffen (BVL 2009d; FiBL 2009). Was dabei selten erwähnt wird: Organische Moleküle natürlicher Provenienz können vergleichbar toxisch wie synthetische Wirkstoffe sein (Ames et al. 1990). Kupfer- und Schwefelpräparate gehören zu den ältesten Pflanzenschutzmitteln überhaupt. Sie werden in allen Landbauformen bei der Bekämpfung von z.B. Mehltaupilzen und Schorf erfolgreich eingesetzt. Der ökologische Landbau bezeichnet Kupfer als unverzichtbar, da sonst ein Totalausfall von Ernten eintreten kann (Das Erste 2008). Diese oft in hohen Aufwandmengen eingesetzte ,alte Chemie“ wirkt unspezifisch und kann deshalb mehr und größere Nebenwirkungen entfalten als neue, selektiv wirkende und abbaubare Pflanzenschutzmittel. Kupfer kann sich im Boden anreichern und toxisch auf Nicht-Zielorganismen, z. B. in Böden und Gewässern, wirken. Vor allem in den Dauerkulturen Wein, Obst und Hopfen sowie in Kartoffeln und einigen Gemüsearten stehen für den Ökolandbau keine hinreichend wirksamen Alternativen zum Kupfer zur Verfügung (Kühne et. al. 2009).

\section{Zusammenfassung und Fazit}

\subsection{Aufklärung der Verbraucher}

Eine mutmaßliche Folge der mangelhaften oder falschen Informationen der Verbraucher zum Thema Pflanzenschutz: Ihre größte Sorge in Bezug auf Ernährung und Gesundheit sind in Deutschland die Pestizidrückstände (Geslain-Lanéelle 2007). Dabei stellt in Wirklichkeit überhöhtes Körpergewicht (neben mikrobiellen Verunreinigungen wie Bakterien und Pilzen in der Nahrung) in der EU eines der größten Pro- bleme bei Ernährung und Gesundheit dar. Die Bedeutung objektiver und wirksamer Aufklärung des Verbrauchers über echte Risiken ist erkannt. Die Sorgen der Verbraucher sind ernst zu nehmen, auch um aus Ängsten keine Panik erwachsen zu lassen (BfR 2008a; Hensel 2007).

\subsection{Wissenschaft als Entscheidungsgrundlage}

Eine von Stimmungen oder anderen Motiven (Bleser 2007) getriebene Entscheidungsfindung auf politischer Ebene und die damit verbundenen Fehlallokationen von Ressourcen sind zu vermeiden, da die Ressourcen dann andernorts fehlen und es dadurch zu wirklichen Schäden kommt (Bhakdi 2007; Gigerenzer 2007; Hensel 2007). Die Wissenschaftlichkeit muss bei der Bewertung von Studien und der Debatte des gesamten Themas „Pflanzenschutz“ wieder stärker als Entscheidungsgrundlage herangezogen werden.

\subsection{Standortnachteil für die Forschung in Europa}

Es ist absehbar, dass die Zahl der in der EU verfügbaren Wirkstoffe infolge der Novellierung der Zulassungsrichtlinie weiter zurückgehen wird. Insbesondere der aus wissenschaftlicher Sicht nicht nachvollziehbare Wechsel zum gefahrenbasierten Bewertungsansatz mit der Festlegung von Ausschlusskriterien wird dazu wesentlich beitragen. Es ist nicht auszuschließen, dass ganze Wirkstoffgruppen vom Markt verschwinden. Zudem besteht erhebliche Rechtsunsicherheit für die Entwickler von Pflanzenschutzmitteln, da die Ausführungsbestimmungen der neuen Gesetze fehlen bzw. unklar oder vage gehalten sind. Eine zügige Verabschiedung der ausstehenden, neuen Regelungen ist aufgrund der Erfahrungen aus der Vergangenheit nicht zu erwarten.

Die europäische Landwirtschaft beklagt, dass ihr Mittel für wichtige Indikationen insbesondere im Obst- und Gemüsebau fehlen. Die Anzahl verfügbarer Mittel ging in den vergangenen 15 Jahren in der EU stark zurück. Dadurch wurde auch die Entwicklung von Resistenzen begünstigt. Die Entwicklung neuer Produkte und Wirkmechanismen ist bei der Lösung dieser Problematik ohne Alternative. Forschung und Innovation können jedoch nur durch geeignete gesetzliche und marktwirtschaftliche Rahmenbedingungen gefördert werden.

\section{Literatur}

Ames BN, Profet M, Gold LS (1990) Nature's chemicals and synthetic chemicals: Comparative toxicology. Proc Natl Acad Sci 87:77827786. http://www.pnas.org/content/87/19/7782.full.pdf+html (Verweis: Juni 2009)

Bach M, Frede HG (2008) Entwicklung der Pflanzenschutzmittel-Frachten in Flussgebieten in Deutschland. Mitt. Julius Kühn Institut 417:304. http://www.bba.de/veroeff/mitt/pdfs/ mitt417.pdf (Verweis: Juni 2009) 
BfR (Bundesinstitut für Risikobewertung) (2008a) Rechtfertigen „gefühlte" Risiken staatliches Handeln? Tagungsband zur Festveranstaltung am 7. November 2007, BfR, Berlin. http://www. bfr.bund.de/cm/238/rechtfertigen_gefuehlte_risiken_staatliches_ handeln tagungsband.pdf (Verweis: Juni 2009)

BfR (Bundesinstitut für Risikobewertung) (2008b) BfR empfiehlt die Beibehaltung der expositionsbasierten Risikobewertung für die Zulassung von Pflanzenschutzmitteln. Information 037/2008, BfR, Berlin. http://www.bfr.bund.de/cm/218/bfr_empfiehlt_die beibehaltung_der_expositionsbasierten_risikobewertung_fuer_die_zulassung_von_pflanzenschutzmitteln.pdf(Verweis: Juni 2009)

BfR (Bundesinstitut für Risikobewertung) (2008c) Analysenergebnisse von PAN Europe: BfR sieht keine gesundheitlichen Risiken durch die nachgewiesenen Pestizid-Rückstände in Wein. Stellungnahme Nr. 012/2008, BfR, Berlin. http://www.bfr.bund.de/cm/218/ analysenergebnisse_durch pan_europe.pdf (Verweis: Juni 2009)

BfR (Bundesinstitut für Risikobewertung) (2008 d) Kontroverse zwischen BfR und Greenpeace über die wissenschaftliche Ableitung sicherer EU-Höchstgehalte von Pflanzenschutzmittelrückständen. Sammlung der Dokumente zu diesem Thema auf der Internetseite des BfR: http://www.bfr.bund.de/cd/27526; http://www.bfr. bund.de/cm/207/veroeffentlichungen_ueber_unsichere_hoechstmengen_von_pestizidrueckstaenden_in_lebensmitteln.pdf (Verweis: Juni 2009)

Bhakdi S (2007) Tatsächliche Risiken - Stiefkinder der Öffentlichkeit? In: Rechtfertigen ,gefühlte" Risiken staatliches Handeln? Tagungsband zur Festveranstaltung am 7. November 2007, BfR, Berlin, S 49-50 http://www.bfr.bund.de/cm/238/rechtfertigen gefuehlte risiken staatliches handeln tagungsband.pdf (Verweis: Juni 2009)

Bleser P (2007) Verbraucherschutz auf Bundesebene. In: Rechtfertigen „gefühlte“ Risiken staatliches Handeln? Tagungsband zur Festveranstaltung am 7. November 2007, BfR, Berlin, S 29-32 http:// www.bfr.bund.de/cm $/ 238 /$ rechtfertigen_gefuehlte_risiken_staatliches_handeln_tagungsband.pdf (Verweis: Juni 2009)

BMELV (Bundesministerium für Ernährung, Landwirtschaft und Verbraucherschutz) (2008) Nationaler Aktionsplan zur nachhaltigen Anwendung von Pflanzenschutzmitteln. Risikominderung im Pflanzenschutz. Weniger Risiko - mehr Vertrauen. Bonn. http://www.bmelv.de/SharedDocs/Downloads/Landwirtschaft/Pflanze/Pflanzenschutz/NationalerAktionsplanPflanzenschutz.html?nn=309982 (Verweis: Juni 2009)

BMELV (Bundesministerium für Ernährung, Landwirtschaft und Verbraucherschutz) (2008) Nationaler Aktionsplan zur nachhaltigen Anwendung von Pflanzenschutzmitteln. Risikominderung im Pflanzenschutz. Weniger Risiko - mehr Vertrauen. Bonn. http://www.bmelv.de/SharedDocs/Downloads/Landwirtschaft/Pflanze/Pflanzenschutz/NationalerAktionsplanPflanzenschutz.html?nn=309982 (Verweis: Juni 2009)

BVL (Bundesamt für Verbraucherschutz und Lebensmittelsicherheit) (2004) Nutzen-Risiko-Abwägung bei der Zulassung von Pflanzenschutzmitteln. Braunschweig. http://www.bvl.bund.de/ cln_007/nn_492010/DE/04_Pflanzenschutzmittel/00_doks downloads/zul_dok_nutzen_risiko,templateId=raw,property =publicationFile.pdf/zul dok nutzen risiko.pdf (Verweis: Juni 2009)

BVL (Bundesamt für Verbraucherschutz und Lebensmittelsicherheit) (2009a) EU-Wirkstoffprüfung. Braunschweig. http://www.bvl. bund.de/cln_007/nn_492010/DE/04_Pflanzenschutzmittel/04 EUWirkstoffpruefung/euwirkstoffpruefung_node.html__ nnn=true (Verweis: Juni 2009)

BVL (Bundesamt für Verbraucherschutz und Lebensmittelsicherheit) (2009b) Wirkstoffprüfung und Zulassung. Braunschweig. http://www.bvl.bund.de/cln_007/nn_492016/DE/04_Pflanzenschutzmittel/01_ZulassungWirkstoffpruefung/zulassung _ node.html_nnn=true (Verweis: Juni 2009)
BVL (Bundesamt für Verbraucherschutz und Lebensmittelsicherheit) (2009c) Liste der zugelassenen Pflanzenschutzmittel. Braunschweig. http://www.bvl.bund.de/cln_007/nn_492000/DE/04 Pflanzenschutzmittel/02_ZugelassenePflanzenschutzmittel/zugelassenePflanzenschutzmittel_node.html_nnn=true (Verweis: Juni 2009)

BVL (Bundesamt für Verbraucherschutz und Lebensmittelsicherheit) (2009d) Liste der zugelassenen Pflanzenschutzmittel für den Ökolandbau. Braunschweig. http://www.bvl.bund.de/cln_007/ nn_492012/DE/04_Pflanzenschutzmittel/00_doks_downloads/psm_oekoliste-DE,templateId=raw,property $=$ publication File.pdf/psm_oekoliste-DE.pdf (Verweis: Juni 2009)

BVL (Bundesamt für Verbraucherschutz und Lebensmittelsicherheit) (2009e) Deutsches Obst und Gemüse hält gesetzliche Höchstgehalte für Pflanzenschutzmittel weitgehend ein. Berlin. http://www. bvl.bund.de/cln_027/nn_493682/DE/08_PresseInfothek/01 Presse und Hintergrundinformationen/01 PI und H $\overline{\mathrm{HI} /}$ Rueckstaende/2009/pi_nb_psm_rueckstaende_2007.html (Verweis: Juni 2009)

BVL (Bundesamt für Verbraucherschutz und Lebensmittelsicherheit) (2009f) Nationale Berichterstattung Pflanzenschutzmittel-Rückstände 2007. Braunschweig. http://www.bvl.bund.de/cln_027/ nn_493682/DE/01_Lebensmittel/01_Sicherheit_Kontrollen/ 05 NB PSM Rueckstaende/01 nb psm/nbpsm 2007/ nbpsm_Bericht_2007.html (Verweis: Juni 2009)

Das Erste - Plusminus (2008) Kupfereinsatz: Biobauern in der Zwickmühle.ARD/NDR, Hamburg, Kurzfassung in Textform: http://www. daserste.de/plusminus/beitrag_dyn uid,ccedp1aamjcmc $9 \mathrm{gj} \sim \mathrm{cm}$. asp, http://www.ardmediathek.de/ard/servlet/content/3107186(Verweis: Oktober 2009)

EFSA (2008) Scientific Opinion of the Panel on Plant protection products and their Residues on a request from the EFSA PRAPeR Unit on risk assessment for birds and mammals. EFSA J 734:1-181 http://www.efsa.europa.eu/EFSA/efsa locale-1178620753812 1211902014630.htm (Verweis: Juni 2009)

FiBL (Forschungsinstitut für Biologischen Landbau) (2009) Betriebsmittelliste für Deutschland, Hilfsstoffliste für den biologischen Landbau in der Schweiz. Frick https://www.fibl-shop.org/shop/ pdf/1032-hilfsstoffliste.pdf (Verweis: Juni 2009)

Geslain-Lanéelle C (2007) Der Sicherheit der europäischen Lebensmittel verpflichtet. In: Rechtfertigen ,gefühlte“ Risiken staatliches Handeln? Tagungsband zur Festveranstaltung am 7. November 2007, BfR, Berlin, S 19-22 http://www.bfr.bund.de/cm/238/ rechtfertigen_gefuehlte_risiken_staatliches_handeln_tagungsband.pdf (Verweis: Juni 2009 )

Gigerenzer G (2007) Ursachen gefühlter Risiken. In: Rechtfertigen „gefühlte" Risiken staatliches Handeln? Tagungsband zur Festveranstaltung am 7. November 2007, BfR, Berlin, S 41-47 http:// www.bfr.bund.de/cm/238/rechtfertigen_gefuehlte_risiken_staatliches_handeln_tagungsband.pdf (Verweis: Juni 2009)

Greenpeace (2002) Bio-Lebensmittel - die sichere Alternative. Greenpeace Redaktion, Hamburg. http://www.greenpeace.de/themen/ landwirtschaft/alternativen/artikel/bio_lebensmittel_die_sichere_alternative/ (Verweis: Juni 2009)

Greenpeace und Global 2000 (2008) Die unsicheren Pestizidhöchstmengen in der EU. Greenpeace und Global 2000, Hamburg Wien. http://www. greenpeace.de/fileadmin/gpd/user_upload/themen/umweltgifte/EU_ Pestizidhoechstmengen270808 AT.pdf (Verweis: Juni 2009)

Greim H (2007) Folgen „gefühlter“ Risiken - Erfahrungen und Erwartungen - Wissenschaft. In: Rechtfertigen ,gefühlte“ Risiken staatliches Handeln? S 86, Tagungsband zur Festveranstaltung am 7. November 2007, BfR, Berlin, S 85-89 http://www.bfr.bund.de/ $\mathrm{cm} / 238 /$ rechtfertigen_gefuehlte risiken_staatliches handeln tagungsband.pdf (Verweis: Juni 2009)

Gutsche V, Strassemeyer J (2007) SYNOPS - ein Modell zur Bewertung des Umwelt-Risikopotentials von chemischen Pflanzenschutz- 
mitteln. Nachrichtenbl Deut Pflanzenschutzd 59(9):197-210 Auszüge unter: http:/www.bmelv.de/SharedDocs/Downloads/ Landwirtschaft/Pflanze/Pflanzenschutz/NationalerAktionsplanPflanzenschutz.html?nn=309982 (S 24 f.) (Verweis: Juli 2009)

Hensel A (2007) Wissenschaft in der Gesellschaft - Wissenschaft für die Gesellschaft: Wem kann man heute noch glauben? In: Rechtfertigen ,gefühlte“ Risiken staatliches Handeln? Tagungsband zur Festveranstaltung am 7. November 2007, BfR, Berlin, S 33-39 http://www.bfr.bund.de/cm/238/rechtfertigen_gefuehlte_risiken staatliches_handeln_tagungsband.pdf(Verweis: Juni 2009)

Kirchmann H, Bergström L, Kätterer T, Andrén O, Andersson R (2009) Can Organic Crop Production Feed the World? In: Kirchmann H, Bergström L (eds) Organic Crop Production - Ambitions and Limitations, Springer, Dordrecht, pp 39-72 http://pub-epsilon. slu.se/508/, http://www.springerlink.com/content/978-1-40209315-9 (Verweis: Juli 2009)

Krautter M (2009) Pestizide in Obst und Gemüse. Probleme und Lösungen für den Verbraucher- und Umweltschutz. Niedersächsischer Landtag, Hannover. http://www.manfredkrautter. de/mediapool/81/819129/data/Praesentation_Landtag_Hannover_19_Mai_2009_Krautter_Webversion.pdf (Verweis: Juni 2009)

Kühne S, Strassemeyer J, Rossberg D (2009) Anwendung kupferhaltiger Pflanzenschutzmittel in Deutschland. J Kulturpfl 61(4): $126-130$
Küster H (2009) Schöne Aussichten - kleine Geschichte der Landschaft. C. H. Beck, München

Oerke EC, Dehne HW (1997) Global crop production and the efficacy of crop protection - current situation and future trends. Europ J Plant Pathol 103:203-215

PSD (Pesticide Safety Directorate) (2008) Revised assessment of the impact on crop protection in the UK of the „cut-off criteria“ and substitution provisions in the proposed Regulation of the European Parliament and of the Council concerning the placing of plant protection products on the market. http://www.pesticides. gov.uk/uploadedfiles/Web_Assets/PSD/Revised_Impact_Report_1_Dec_2008(final).pdf (Verweis: Juni 2009)

Positivliste (Anhang I) der Richtlinie 91/414/EEC. http://ec.europa.eu/ food/plant/protection/evaluation/dir91-414eec_en.htm (Verweis: Juni 2009)

Schmidt B, Neuffer T, Häfner M, Dechet F, Küchler T, Peters B, Resseler H, von Götz N (2005) Evaluation of Plant Protection Product Findings in Groundwater in Germany. Nachrichtenbl Deut Pflanzenschutzd 57(11):213-223 http://www.iva.de/pdf/De_Grundwasserstudie_011105.pdf (Verweis: Juni 2009)

SRU (Sachverständigenrat für Umweltfragen) (2004) Umweltgutachten 2004 des Rates von Sachverständigen für Umweltfragen. Drucksache 15/3600, Deutscher Bundestag, Berlin. http://www. umweltrat.de/02gutach/downlo02/umweltg/UG_2004.pdf (Verweis: Juni 2009) 